EPJ Web of Conferences 92, 02022 (2015)

DOI: 10.1051 /epjconf/ 20159202022

(C) Owned by the authors, published by EDP Sciences, 2015

\title{
Evaluation of pump characteristic from measurement of fast deceleration
}

Daniel Himr ${ }^{1, \text { a }}$ and Vladimír Habán ${ }^{2}$

1 VŠB - Technical University of Ostrava, Faculty of Mechanical Engineering, Department of Hydrodynamics and Hydraulic Equipment, 17. listopadu 15, 708 33, Ostrava, Czech Republic

2 Brno University of Technology, Faculty of Mechanical Engineering, Energy Institute, Victor Kaplan Department of Fluid Engineering, Technická 2, 616 69, Brno, Czech Republic

\begin{abstract}
Article describes an experiment where a pump connected to the simple hydraulic circuit is decelerated. Since the deceleration is fast enough the operating point of the machine moves from the initial steady position to the breaking zone, turbine zone and back to the new steady position. A dependence of the specific energy and the torque on the flow rate was evaluated from the measurement of the input and output pressure, torque and rotational speed recorded during the deceleration. Obtained characteristic is much wider than curves obtained from regular measurement of steady state.
\end{abstract}

\section{Introduction}

Pipeline transients are often connected with pump transients when the machine is starting or stopping. Pump does not operate under steady condition during these events and operating point can go out of the pumping regime. When one designs a pumping system it is necessary to consider such transients and suggest appropriate protection of pipeline system.

A sudden pump trip is a typical example of potentially dangerous event [1]. Energy, which pump supplies to a hydraulic system, is given by pump characteristic. It depends on the flow rate and rotational speed. When pump loses input power its speed goes down according to current flow rate and torque characteristic. Energy supplied by pump goes down as well as speed and can reach a high negative value which brings a risk of cavitation.

To predict the pump behaviour during the stopping period or the black out, it is necessary to know the extended pump characteristic. Producer usually does not provide this characteristic because it is difficult and expensive to obtain it. This paper describes a procedure how to get the extended characteristic from measurement of fast pump deceleration in a simple hydraulic circuit. There is a lot of articles dealing with the problem of pump deceleration [2], [3] or acceleration [4], [5], [6], but authors found only little information about reconstruction of pump characteristic from measurement of this transients.

\section{Theory}

The simplest circuit for measurement of pump characteristic is shown in the figure 1. It consists of a tank, measured pump and a valve, which serves for flow rate regulation. For evaluation of the characteristic, one has to measure input pressure $p_{2}$, output pressure $p_{3}$, flow rate $Q$, speed $n$ and torque $T$. The last two items are gained by dynamometer.

\footnotetext{
a e-mail: daniel.himr@vsb.cz
}

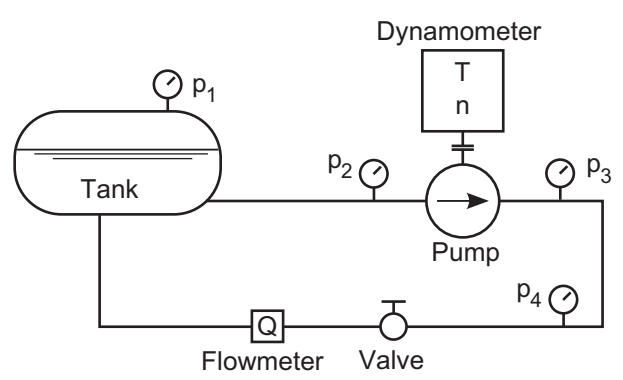

Fig. 1. Experimental set-up

Procedure of the measurement is following: To start the pump, to set constant speed and to open the valve as much as possible. When flow rate stabilizes, all measured quantities can be write. Then, reduction of the flow rate with the valve follows and measurement of the new values of measured quantities can be done. These steps are repeated till the flow rate is zero. Only short part of the whole pump characteristic can be obtained by this procedure. It is plotted by solid lines in the figure 2. Maximal flow rate in the

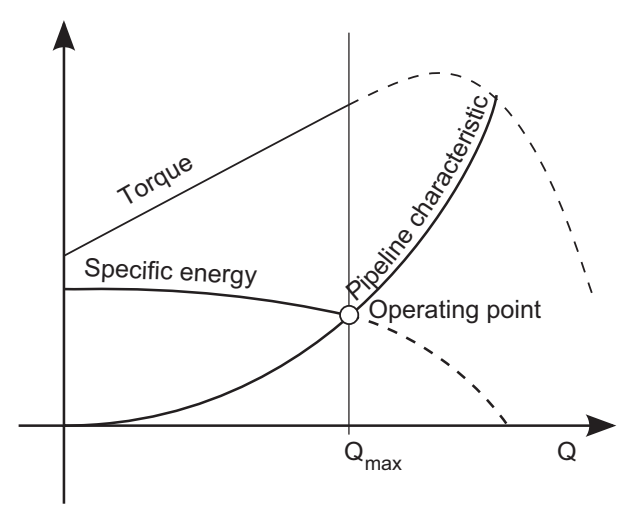

Fig. 2. Operating point of the system, when the valve is fully open [7]

system is limited by minimal possible pipeline resistance. 
But the pump characteristic continues over this point and torque curve changes significantly in this zone. The knowledge of it is important when one needs to simulate pump trip in the system with great inertia and low head [8].

The testing circuit should contain another pump to get over the limit $Q_{\max }$ and to obtain accurate measurement of the extended characteristic.

There is another possibility, which does not involve two pumps in the circuit: When tested pump gives maximal flow rate and its shaft is suddenly slowed down, the pump characteristic changes according to affinity law, but the inertia of the water column keeps flow rate the same at the first moment. Operating point moves to the new characteristic and, after certain time, finds a new steady position given by intersection of the new pump characteristic and pipeline characteristic. The process is shown in the figure 3 .

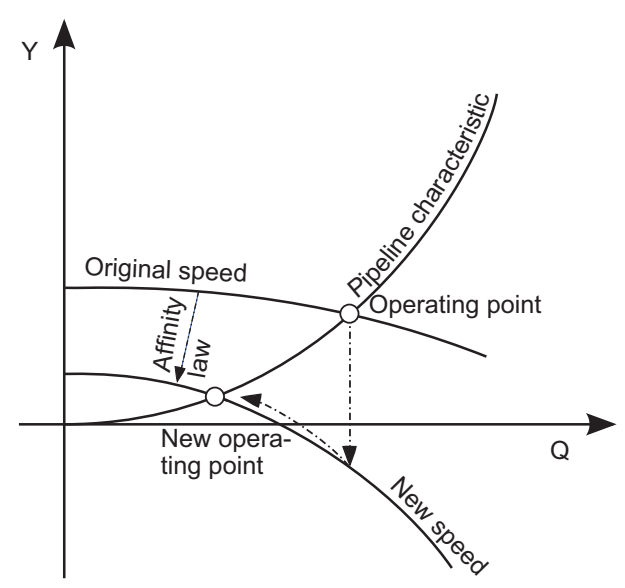

Fig. 3. Movement of the operating point while the pump is decelerating

Equations (1) to (4) describe the affinity law. Subscript $o$ means original value.

$$
\begin{aligned}
\frac{Q_{o}}{Q} & =\frac{n_{o}}{n}, \\
\frac{Y_{o}}{Y} & =\left(\frac{n_{o}}{n}\right)^{2}, \\
\frac{T_{o}}{T} & =\left(\frac{n_{o}}{n}\right)^{2}, \\
\frac{\eta_{o}}{\eta} & =1 .
\end{aligned}
$$

The measurement of this unsteady process and following evaluation differ from measurement of the steady state.

\section{Measurement}

Parameters of tested pump are listed in the table 1. It is a centrifugal pump made of steel (impeller) and cast iron (spiral case), which was connected to the circuit according to figure 1 . The pressure was measured with pressure transducers (range $0-160 \mathrm{kPa}$ abs. for suction and $0-400 \mathrm{kPa}$ abs. for discharge, accuracy $0.25 \%$ of the range) in four places marked in the figure. Electromagnetic flow meter was used just for steady flow (range $0-5001 / \mathrm{s}$, accuracy
Table 1. Pump specifications

\begin{tabular}{lll}
\hline Entry & Value & Unit \\
\hline Suction diameter & 0.39 & $\mathrm{~m}$ \\
Discharge diameter & 0.352 & $\mathrm{~m}$ \\
Impeller diameter & 0.41 & $\mathrm{~m}$ \\
Number of vanes & 6 & - \\
Specific speed & 310 & $\mathrm{rpm}$ \\
\hline
\end{tabular}

$0.2 \%$ of measured value), dynamometer controlled speed, measured torque (range $0-1000 \mathrm{Nm}$, accuracy $0.5 \%$ of measured value) and revolutions (range $0-4500 \mathrm{rpm}, 1000$ pulses per revolution). As the sampling frequency of revolution measurement was $10 \mathrm{~Hz}$ all other quantities were measured with the same frequency.

Measurement of steady characteristic was the first step. Figure 4 is valid for speed $n_{0}=1000 \mathrm{rpm}$, which was chosen as a reference value. The characteristic ends almost immediately behind the best efficiency point due to high resistance of the hydraulic circuit.

All parameters with subscript opt mean the best efficiency point for speed $1000 \mathrm{rpm}$.

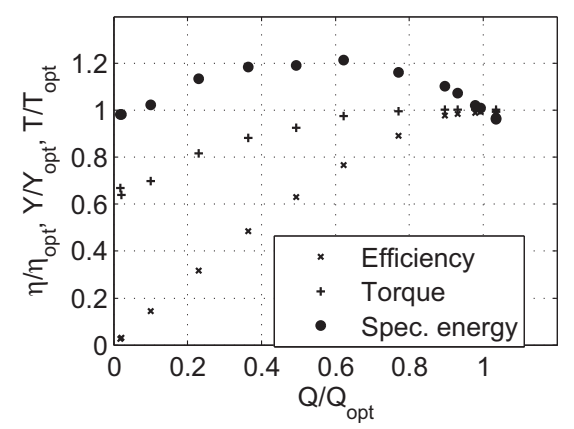

Fig. 4. Characteristic of the pump

\subsection{Moment of inertia}

To obtain characteristic from pump deceleration, the inertia moment of the pump is necessary. It was found from deceleration when the discharge was closed. It means that torque was known from static characteristic $(Q=0)$ and equation (3). Initial speed $1140 \mathrm{rpm}$ was reduced to $10 \mathrm{rpm}$ in $5.65 \mathrm{~s}$, it is deceleration $200 \mathrm{rpm}$ per second. Graph in the figure 5 was plotted using equation 5 , where measured torque $T_{m}$ is reduced by the value $T(Q=0, n)$. The speed changed linearly between values $1000 \mathrm{rpm}$ and $100 \mathrm{rpm}$, so the inertia $\left(9.5 \mathrm{~kg} \cdot \mathrm{m}^{2}\right)$ was evaluated from this range. The result was the same for any deceleration.

$$
I=\frac{\left(T_{m}-T\right) \Delta t}{2 \cdot \pi \cdot \Delta n}
$$

Potentially, the same process can be applied when pump is accelerated, but the results are not unambiguous.

\subsection{Flow rate}

Unsteady flow rate cannot be measured with the electromagnetic flowmeter, because this device has a great inte- 


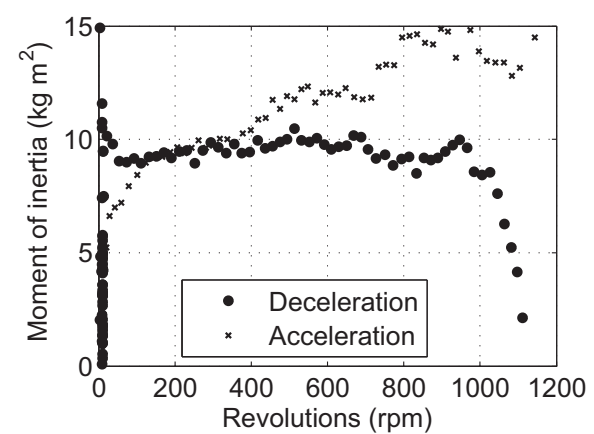

Fig. 5. Moment of inertia from pump acceleration and deceleration

Table 2. Numerical model parameters

\begin{tabular}{llll}
\hline Entry & Suction & Discharge & Unit \\
\hline Length & 1.65 & 8 & $\mathrm{~m}$ \\
Diameter & 0.39 & 0.352 & $\mathrm{~m}$ \\
Roughness & 0.5 & 0.5 & $\mathrm{~mm}$ \\
Viscosity & $10^{-6}$ & $10^{-6}$ & $\mathrm{~m}^{2} / \mathrm{s}$ \\
Density & 1000 & 1000 & $\mathrm{~kg} / \mathrm{m}^{3}$ \\
Wave speed & 1000 & 1000 & $\mathrm{~m}$ \\
Length step & 0.55 & 0.5 & $\mathrm{~m}$ \\
Courant number & 1 & 1 & - \\
\hline
\end{tabular}

grating constant so it is not suitable for unsteady events. To get over this complication, it is possible to compute flow rate as a function of time from pressure measured in two places. In our case, inlet flow rate was computed from pressures $p_{1}$ and $p_{2}$ and outlet flow rate was evaluated from pressures $p_{3}$ and $p_{4}$, see figure 1 .

The flow rate was obtained as a solution of equations (6, 7) with known pressure boundary conditions $p_{1}$ and $p_{2}$ for inflow and $p_{3}$ and $p_{4}$ for outflow.

$$
\begin{aligned}
\frac{\partial Q}{\partial t}+\frac{S}{\rho} \frac{\partial p}{\partial x}+\frac{\lambda}{2 D S}|Q| Q & =0 \\
\frac{\partial p}{\partial t}+\frac{K}{S} \frac{\partial Q}{\partial x} & =0
\end{aligned}
$$

The Lax-Wendroff numerical scheme was used here. Basic parameters of the numerical model are listed in the table 2, where length means distance between corresponding pressure transducers. Coefficient of friction loss was obtained by Churchill's relationship. Figure 6 shows an example of computed flow rate. One can see that suction flow rate and discharge flow rate are almost identical.

Simpler method how to find flow rate from pressure difference is known as Gibson's method. It can be derived by integration of equation (6), see relationship (8).

$$
Q(t+\Delta t)=Q(t)-\frac{\Delta t S}{L \rho}[\Delta p(t)+R|Q(t)| Q(t)],
$$

where $L$ is a distance between pressure transducers and $\Delta p$ is their pressure difference. This equation is subject to numerical iteration as the resistance $R$ is a function of initial flow rate. Compare the result in the figure 7 with the figure 6. It only slightly differs, graphs are comparable. This approach is possible, when the pressure does not go too deep under atmospheric pressure, thus the fluid density is constant [9].

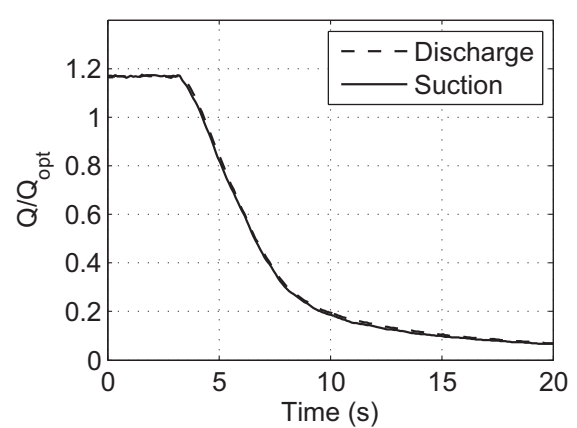

Fig. 6. Flow rate when pump decelerated from speed $1140 \mathrm{rpm}$ to $10 \mathrm{rpm}$. Deceleration rate: $266.67 \mathrm{rpm}$ per second

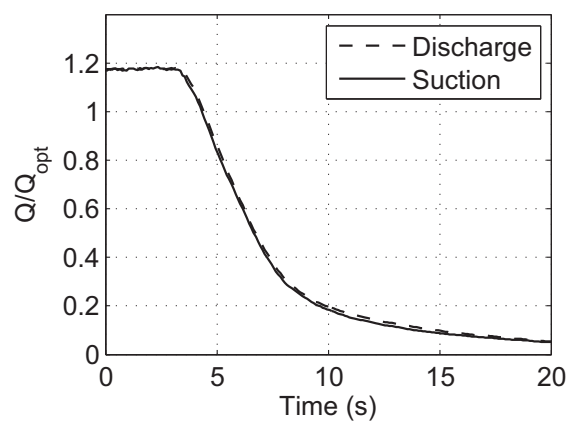

Fig. 7. Flow rate when pump decelerated from speed $1140 \mathrm{rpm}$ to $10 \mathrm{rpm}$. Deceleration rate: $266.67 \mathrm{rpm}$ per second. (Gibson method)

\section{Pump characteristic}

We evaluated pump characteristic from seven different decelerations. Initial speed was always $1140 \mathrm{rpm}$ (maximal possible speed with respect to input power) and the final speed was $10 \mathrm{rpm}$ (the lowest possible speed of dynamometer measurement). Corresponding initial flow rate was $1.175 \cdot Q_{\mathrm{opt}}$ and four deceleration rates were measured: $266.67 ; 400.00 ; 571.42$ and $800.00 \mathrm{rpm}$ per second. We also measured three cases with initial flow rate equal $Q_{\text {opt }}$ (but the initial speed was still $1140 \mathrm{rpm}$ ) and three deceleration rates $266.67 ; 400.00$ and $800.00 \mathrm{rpm}$ per second. The last three cases served for check if the characteristic obtained from unsteady event would cover the steady characteristic. All seven cases are plotted in the figure 8 (some of them were measured twice). The figure shows one quarter of Karman-Knapp characteristic.

Places, where the specific energy equals zero, should be on the same half line. The same statement is valid also for places, where the torque is zero (runaway). Both half lines are plotted in the graph as well. They were found by least square method (LSM).

The instant specific energy was computed by equation (9) and instant torque by equation (10).

$$
\begin{aligned}
Y= & \int_{x_{2}}^{x_{3}} \frac{\mathrm{d} Q}{S \mathrm{~d} t} \mathrm{~d} x+\frac{p_{3}-p_{2}}{\rho}+\frac{8 Q^{2}}{\pi^{2}} \cdot\left(D_{3}^{-4}-D_{2}^{-4}\right)+ \\
& +g \cdot\left(H_{3}-H_{2}\right), \\
T= & T_{m}-2 \pi \cdot I \cdot \frac{\Delta n}{\Delta t} .
\end{aligned}
$$




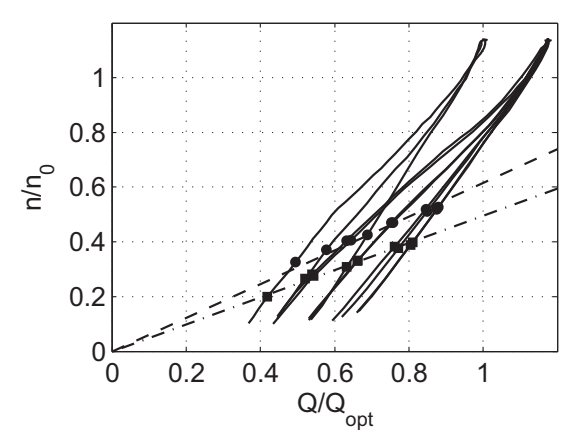

Fig. 8. Measured cases of pump deceleration (solid line), measured zero specific energy (spots), zero specific energy by LSM (dashed line), measured zero torque (squares) and zero torque by LSM (broken line)

Integral limits $x_{2}$ and $x_{3}$ delimit pipe from pressure transducer $p_{2}$ to the impeller inlet and from impeller outlet to pressure transducer $p_{3}$. Spiral case is included. The equations do not consider variable speed. The specific energy is plotted in the figure 9, where one can see that the process was strongly unsteady. Specific energy started from

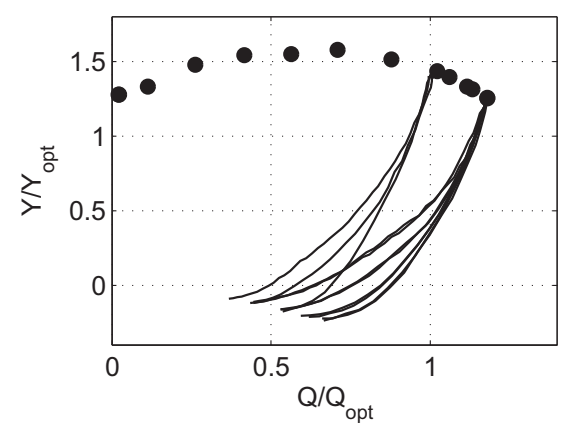

Fig. 9. Specific energy during deceleration (solid lines) compared with the static characteristic (spots)

the static pump characteristic line and reached a negative value in all cases.

The torque is plotted in the figure 10. Again, the initial value was on the static characteristic line and dropped down to the negative value. Only data, where speed was

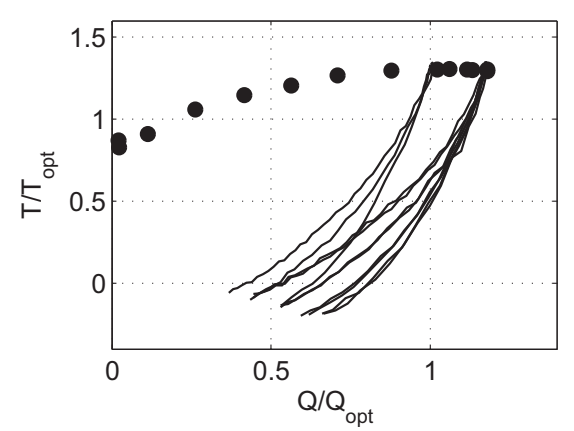

Fig. 10. Torque during deceleration (solid lines) compared with the static characteristic (spots)

greater than $100 \mathrm{rpm}$, were evaluated. Thus all solid lines in the figures 9 and 10 are not complete, because speed under $100 \mathrm{rpm}$ brought too big error.

When the results is corrected by affinity law, see equations $(1-3)$, the extended pump characteristic can be plotted. It is shown in figures 11 and 12.
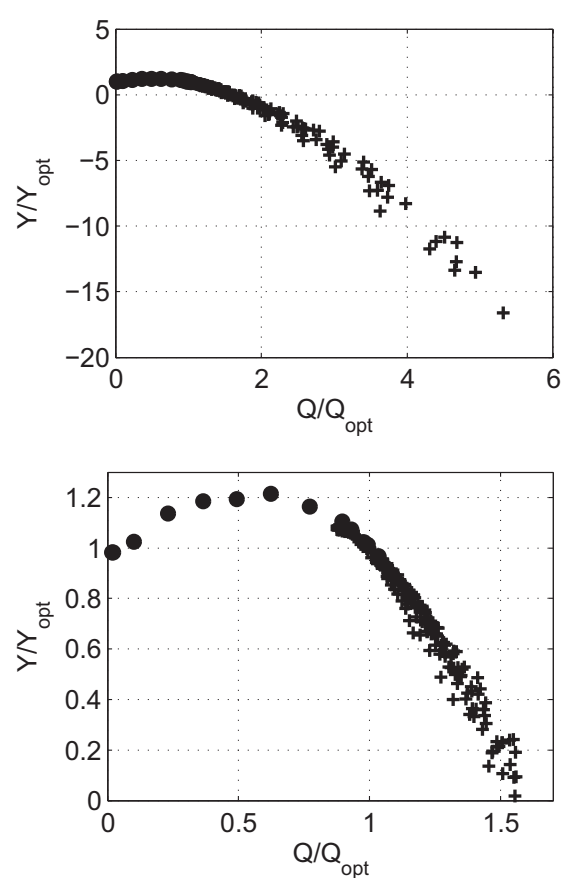

Fig. 11. Evaluated specific energy of the pump for speed $1000 \mathrm{rpm}$. Static characteristic (spots) is extended by dynamic measurement (crosses). Bottom graph is a detail
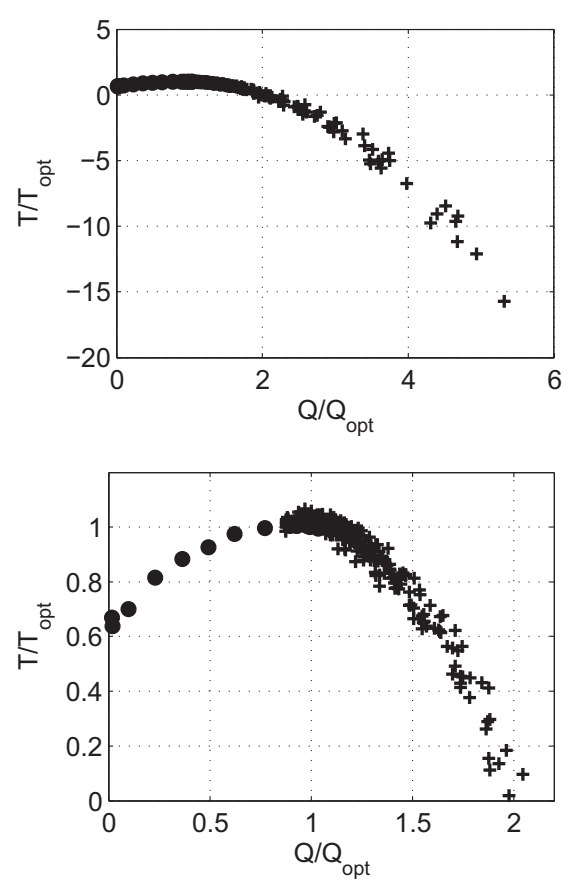

Fig. 12. Evaluated torque of the pump for speed $1000 \mathrm{rpm}$. Static characteristic (spots) is extended by dynamic measurement (crosses). Bottom graph is a detail 
It is obvious that part of characteristic obtained from deceleration is reasonable despite the fact that it is more scattered than part obtained from measurement of steady state. It means that described procedure is good when an approximate characteristic of the pump is needed. Typically, for numerical simulation of transient events in pipeline systems, where the error of several percent is, usually, acceptable. But this procedure cannot replace exact measurement of the characteristic needed for calculation of operational expenses.

The figure 13 shows the efficiency computed from the regression analysis of the specific energy and the torque for speed $1000 \mathrm{rpm}$. The error in the best efficiency point is about $5 \%$, because the interpolating polynomial of the torque slightly overestimates the characteristic in the vicinity of the best efficiency point.

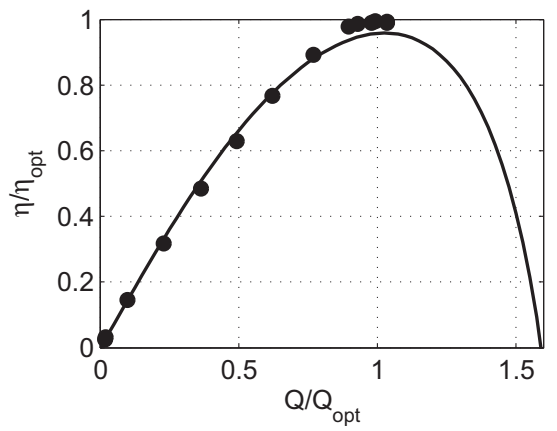

Fig. 13. Measured efficiency from steady state (spots) and regression function from measurement of steady state and deceleration (solid line)

The data, where $Y>0$, were interpolated by polynomial function to get function of specific energy (11) and to get function of torque (12). Both sets of data (obtained by measurement of steady and unsteady state) were used as the input. The figure 13 is plotted according to (13).

$$
\begin{aligned}
\frac{Y}{Y_{o p t}}= & -1.0689 \cdot\left(\frac{Q}{Q_{o p t}}\right)^{2}+1.1081 \cdot \frac{Q}{Q_{o p t}}+ \\
& +0.9448, \\
\frac{T}{T_{o p t}}= & -0.2786 \cdot\left(\frac{Q}{Q_{o p t}}\right)^{3}+0.0647 \cdot\left(\frac{Q}{Q_{o p t}}\right)^{2}+ \\
& +0.5856 \cdot \frac{Q}{Q_{o p t}}+0.6502, \\
\frac{\eta}{\eta_{o p t}}= & \frac{Q}{Q_{o p t}} \cdot \frac{Y}{Y_{o p t}} \cdot \frac{T_{o p t}}{T} .
\end{aligned}
$$

Finally, the flow rate, where the specific energy equals zero, was found as $1.59 \cdot Q_{\mathrm{opt}}$ with the approximate error $\pm 5 \%$. The flow rate, where the torque equals zero, was found as $1.93 \cdot Q_{\mathrm{opt}}$ with the approximate error $\pm 3 \%$. The error is defined from standard deviation.

\section{Conclusion}

The paper describes evaluation of pump characteristic from data obtained during measurement of pump deceleration. The process allows measuring the pump characteristic over the point of maximal flow rate in the hydraulic circuit that is defined by intersection of pump characteristic and pipeline characteristic, thus there is no need to install another pump to the circuit.

The result is not as accurate as characteristic gained by measurement of steady state, but it reaches braking and turbine zones of the machine. This is very useful, because producers, usually, does not provide this parts of characteristic. The result is accurate enough to use it as an input when numerical simulation of pump transient is being done.

\section{Acknowledgement}

This paper was elaborated in the framework of the project Opportunity for young researchers, reg. no. CZ.1.07/2.3.00/ 30.0016, supported by Operational Programme Education for Competitiveness and co-financed by the European Social Fund and the state budget of the Czech Republic.

This paper is also output of NETME Centre, regional $R \& D$ centre built with the financial support from the Operational Programme Research and Development for Innovations within the project NETME Centre (New Technologies for Mechanical Engineering), Reg. no. CZ.1.05/2.1.00/ 01.0002 and, in the follow-up sustainability stage, supported through NETME CENTRE PLUS (LO1202) by financial means from the Ministry of Education, Youth and Sports under the "National Sustainability Programme I."

\section{Nomenclature}

$\begin{array}{lll}D & (\mathrm{~m}) & \text { Diameter } \\ g & \left(\mathrm{~m} \cdot \mathrm{s}^{-2}\right) & \text { Gravitational acceleration } \\ H & (\mathrm{~m}) & \text { Elevation } \\ I & \left(\mathrm{~kg} \cdot \mathrm{m}^{2}\right) & \text { Moment of inertia } \\ K & (\mathrm{~Pa}) & \text { Bulk modulus } \\ L & (\mathrm{~m}) & \text { Length } \\ n & (\mathrm{rps}) & \text { Rotational speed } \\ p & (\mathrm{~Pa}) & \text { Pressure } \\ Q & \left(\mathrm{~m}^{3} \cdot \mathrm{s}^{-1}\right) & \text { Discharge } \\ R & \left(\mathrm{~kg} \cdot \mathrm{m}^{-7}\right) & \text { Resistance } \\ S & \left(\mathrm{~m}^{2}\right) & \text { Pipe cross-section } \\ T & \left(\mathrm{~N} \cdot \mathrm{m}^{2}\right) & \text { Torque } \\ t & (\mathrm{~s}) & \text { Time } \\ x & (\mathrm{~m}) & \text { Longitudinal coordinate } \\ Y & \left(\mathrm{~J} \cdot \mathrm{kg}^{-1}\right) & \text { Specific energy } \\ \Delta n & (\mathrm{rps}) & \text { Speed change } \\ \Delta p & (\mathrm{~Pa}) & \text { Pressure difference } \\ \Delta Q & \left(\mathrm{~m} \cdot \mathrm{s}^{-1}\right) & \text { Discharge change } \\ \Delta t & (\mathrm{~s}) & \text { Time step } \\ \eta & (-) & \text { Efficiency } \\ \lambda & (-) & \text { Coefficient of friction loss } \\ \rho & \left(\mathrm{kg} \cdot \mathrm{m}^{-3}\right) & \text { Density } \\ & & \end{array}$

\section{Subscripts:}

m Measured value

o Original (reference) value

opt Value at the best efficiency point (for $1000 \mathrm{rpm}$ ) 


\section{References}

1. S. Pejovic, A. P. Boldy, Guidlines to Hydraulic Transient Analysis of Pumping Systems (P \& B Press, Belgrade; Coventry 1992), pp. 181

2. H. Tsukamoto, S. Matsunaga, H. Yoneda, S. Hata, Journal of Fluids Engineering 108 (1986), pp. 392 - 398

3. J. Liu, Z. Li, L. Wang, L. Jiao, Journal of Fluids Engineering 133 (2011), pp. 1 - 7

4. H. Tsukamoto, S. Ohashi, Journal of Fluids Engineering 104 (1982), pp. 6 - 13

5. K. Farhadi, A. Bousbia-salah, F. D'Auria, Progress in Nuclear Energy 49 (2007), pp. 499 - 510

6. F. F. Hu, X. D. Ma, D. Z. Wu, L. Q. Wang, IOP Conference Series: Earth Environtal Science 15042016 (2012), pp. 8, DOI: $10.1088 / 1755-1315 / 15 / 4 / 042016$

7. D. Himr, V. Habán, The 22nd International Conference on Hydraulics and Pneumatics (Prague 2013), pp. 91 97), ISBN 978-80-248-3136-7

8. D. Himr, EPJ Web of Conferences 6702035 (2014), pp. 6, DOI: 10.1051/epjconf/20146702035

9. J. Jablonská, EPJ Web of Conferences 6702048 (2014), DOI: $10.1051 /$ epjconf/20146702048 